\title{
Series Chain-link Modular Multilevel ac/dc Converter (SCC) for HVDC Applications
}

\author{
Francesco Tardelli, Member, IEEE, Alessandro Costabeber, Member, IEEE, David Trainer and Jon Clare, Senior \\ Member, IEEE
}

\begin{abstract}
Introduction of the Modular Multilevel Converter (MMC) has enabled the exploitation of Voltage Source Converters (VSCs) in an increasing number of High Voltage Direct Current (HVDC) applications. Subsequently, some new topologies and solutions have been presented to tailor the MMC concept to specific uses. Particular attention has been paid to reduction of the converter footprint for applications where plant size is a critical economic aspect, for example, in off-shore installations.

This paper introduces a new series connected modular multilevel ac/dc converter, the Series Chain-link Converter (SCC), which gives a significant reduction in the required number of submodules (SMs) and a more compact distribution of the energy storage, compared to an MMC. In the paper, the operating principle of the converter and its design are discussed in detail; the sub-module count and energy storage requirement are also given. The basic control loops required for the practical operation of the converter are presented and designed. The SCC concept has been experimentally validated on a small-scale $450 \mathrm{~V}$ dc, $415 \mathrm{~V}$ ac, $4.5 \mathrm{kVA}$ laboratory prototype, confirming the practical viability of the topology.
\end{abstract}

Index Terms-HVDC, Modular Multilevel Converter, Series Chain-link Converter.

\section{INTRODUCTION}

H $\mathrm{Vdc}$ has proven to be a more convenient solution than conventional ac systems for long distance power transmission since the 1950s. Recently, Voltage Source Converters (VSCs) have been extensively investigated as a replacement for Line Commuted Converters (LCCs) in order to (1) reduce the converter station footprint, (2) provide independent control of the active and the reactive power and (3) enable blackstart capabilities. VSC technology has been employed for a long time in medium voltage (MV) applications, such as MV drives [1], [2]. However, the basic 2-level VSC has severe drawbacks at higher voltage and power ratings because of the static and dynamic balancing required for series connected semiconductors, the high switching loss and high $d v / d t$ which impacts on the insulation of transformers and inductors [3]. To overcome the limitations of the 2-level VSC, multilevel conversion has been investigated since the 1980s, starting from the 3-level NPC converter introduced by Nabae et al. in [4]. However, the non-modular structure has limited the application of the NPC in high voltage converters with more

F. Tardelli, J. Clare and D. Trainer are with the Faculty of Engineering, The University of Nottingham, Nottingham NG7 2RD, UK, e-mail: jon.clare@nottingham.ac.uk

A. Costabeber is with the University of Padova, 35131 Padova, ITALY and is an Associate Member of the Faculty of Engineering at the University of Nottingham, UK

This work was supported in part by EPSRC under grant EP/R002924/1. levels. Another solution, the Cascaded H-Bridge (CHB) was proposed in [5] where the multilevel voltages are obtained by the series connection of H-bridges, each requiring an isolated dc source. The first multilevel converter targetted for HVDC applications was the Flying Capacitor converter (FC) [6] but the large number of capacitors, with progressively increasing voltage ratings, required to achieve suitable voltage levels has affected its practical exploitation.

A remarkable step forward in multilevel conversion was presented in [7], where a chain-link of H-bridges with floating capacitors was used in a three-phase STATCOM. In the early 2000s, Marquadt et al. introduced the first Modular Multilevel Converter (MMC) for ac/dc conversion [8]. The MMC has allowed the exploitation of VSC concepts in HVDC applications where compact converter size, black start capability and independent active and reactive power control are required. As highlighted in [9], the footprint of an MMC-VSC converter substation can potentially be between one third and one quarter of an equivalent LCC substation. In addition, the MMC features straightforward redundancy, modular structure, lower power losses than other VSCs and low dv/dt impressed on magnetic components.

The MMC has been adopted in a number of HVDC installations from the main industrial leaders as in [9] and [10]. In off-shore applications, the converter platform plays a key role in the final cost [11], and the advantage of the MMC is the significant reduction in filtering components and the absence of reactive power compensation components. However, improving the MMC power density is one of the most important targets for further developments of the topology. For example, a Twin Module (TM) could be used to compact 3 voltage levels in a single sub-module unit [12].

The constant attention on the MMC concept has led to the introduction of new modular multilevel topologies. In [13] and [14], a hybrid modular multilevel VSC and its augmented version, the Series Bridge Converter (SBC), have been presented. Due to the series connection of phase chainlinks on the dc side and the production of rectified ac waveforms, these topologies have a low number of SMs and reduced energy storage. However, three "unfolding bridges" are required and the control of the ac and dc sides is not completely decoupled. In the Alternate Arm Converter [15], the reduction of SMs is achieved by switching the ac current path from the upper to the lower arms of each phase leg. This also reduces the required energy storage. Despite these advantages, the converter balancing procedure is complicated when the converter is operated away from the "sweet spot". 
The Active Flying Capacitor MMC (AFC-MMC) [16] presents operation similar to the AAC but it uses only one chain-link of SMs per-phase, further reducing the required number of SMs. However, as in the AAC it requires a capacitive filter on the dc side and additionally a series connection of basic commutation cells (IGBT + anti-parallel diode) across the dc link, one per phase. A series connected MMC (SC-MMC) is presented in [17]. As for the MMC the SC-MMC retains decoupled control of the ac and dc side but it requires 12 arm inductors. An additional multilevel topology that uses the series connection of the phases is presented in [18]. Despite the interesting connection this topology requires high energy stored in the chain-links connected in the path of the ac current.

The introduction of the Series Chain-link Converter (SCC) proposed in this paper is motivated by the aim to reduce the number of required sub-modules and to achieve a more compact distribution of the energy within the converter with respect to an MMC, and for this reason it is based on series connection of the converter phases on the dc side. At the same time, the converter is conceived to retain the important characteristics of the MMC such as independent dc and ac control capability, low power losses and similar total energy storage requirements. The design should also guarantee converter operation in abnormal grid scenarios, such as voltage imbalances and more onerous fault conditions.

The rest of the paper is organised as follows. Section II describes the SCC in detail, including the different topological options and the considerations that led to the choice of the final topology of the converter. The basic steady-state equations governing the converter operation are presented, and sizing of the converter is discussed including the number of required sub-modules and energy storage. Section III discusses the control loops and their design. Section IV describes the experimental prototype used for the evaluation of the converter and Section V shows experimental results in steady-state and during transients, confirming the practical feasibility of the topology.

\section{The Series Chain-Link Converter}

The general schematic of the proposed converter is presented in Fig. 1. The SCC is obtained by connecting three longitudinal chain-links (L-CH) of half-bridge sub-modules in series on the dc side. In normal steady state conditions, each $\mathrm{L}-\mathrm{CH}$ must generate one third of the total dc voltage plus the ac voltage of the corresponding phase. The sum of the three L$\mathrm{CH}$ voltages is equal to the total dc link voltage, since in steady state the three ac components in each L-CH are a symmetrical set of voltages. The dc voltage can then be adjusted by a coordinated action on the dc component produced by each L$\mathrm{CH}$. On the ac side, the phase voltage is derived from each L-CH by eliminating the dc "offset". This task is carried out, for each phase, by a transverse branch (T-branch), and the methods proposed to eliminate the dc offset use a combination of either full-bridge or half bridge SMs and passive elements such as inductors and capacitors. The resulting ac voltages are applied to the secondary windings of either three independent single phase transformers or a three phase transformer with all

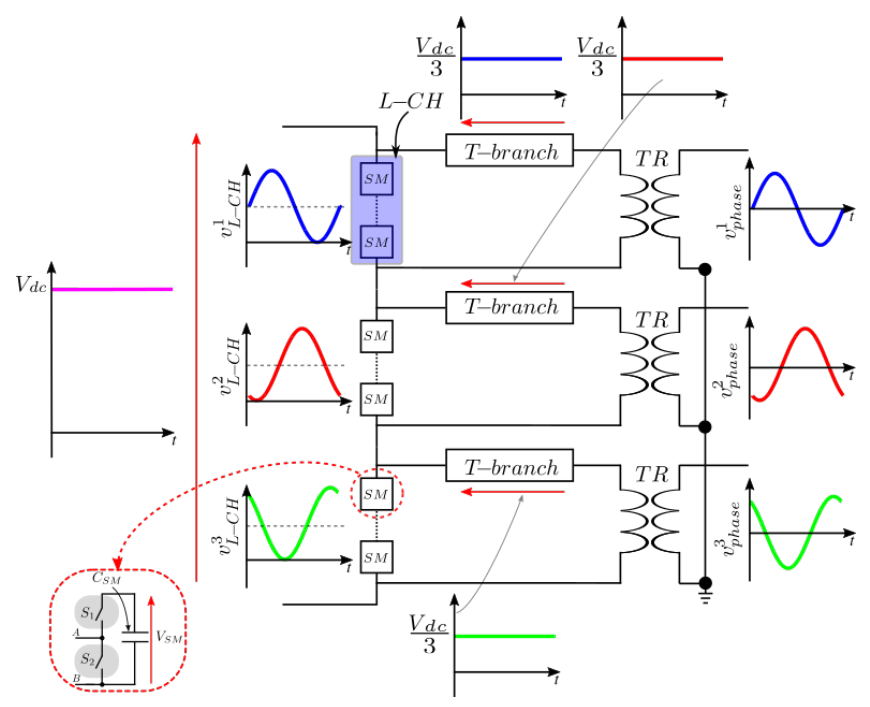

Fig. 1: Schematic of the Series Chain-link Converter (SCC) including ideal voltage waveforms on the ac and dc side of the converter.

the individual winding terminals accessible on the converter side. The three transformer windings on the grid side may be arranged in a star or delta configuration. The need for a transformer is not generally a disadvantage since, although the MMC has been proposed as a transformer-less solution [19], in practice a transformer is nearly always used in HVDC applications to meet system requirements. A transformer is normally required in order to adjust the ac voltage on the converter side [20]. A delta connection of the transformer on the converter side also allows optimisation of the output voltage capabilities of converter by using the third harmonic injection as reported in [21]. The main benefits of using a transformer in an HVDC system are summarised in [22]. The transformer provides additional coupling reactance, adjusts the ac voltages at optimal levels, provides galvanic isolation and prevents the flow of the zero sequence between the ac system and the converter.

\section{A. Converter description}

Despite the different options for the realisation of the Tbranch, which are discussed later, each of the proposed solutions has the same circuit configuration on the dc side, with half-bridge SM L-CHs. To introduce the working principle of the converter, the dc side is considered first. The arrangement in Fig. 2, representing the most general realisation of the Tbranch using a combination of passive and active elements, can be used as a reference for the analysis. For now, the T-branches can be considered as ideal elements that perfectly remove the dc offset produced by the corresponding L-CHs. Assuming the L-CHs are represented as ideal controllable voltage sources, the basic equations describing the converter can be formulated.

During normal operation, the voltage generated by each L$\mathrm{CH}$ circuit is $1 / 3^{\text {rd }}$ of the total de voltage plus the ac voltage component needed to regulate the power transfer between the 
converter and the ac grid. During normal operation, the i-th $(i=1,2,3) \mathrm{L}-\mathrm{CH}$ produces a voltage of the form:

$$
v_{L-C H}^{i}(t)=\frac{V_{d c}}{3}+r \cdot \hat{V}_{\text {phase }} \sin \left(\omega_{g} \cdot t-\frac{i-1}{3} \pi\right)
$$

The current flowing in the i-th L-CH can be described by the following equation,

$$
i_{L-C H}^{i}(t)=I_{d c}-\frac{1}{r} \hat{I}_{\text {phase }} \sin \left(\omega_{g} \cdot t-\varphi-\frac{i-1}{3} \pi\right)
$$

where $\hat{V}_{\text {phase }}$ and $\hat{I}_{\text {phase }}$ are respectively the ac grid voltage and current amplitudes, $\omega_{g}$ is the angular frequency of the grid, $\varphi$ is the phase shift between the ac voltage and the ac current and $r$ is the secondary to primary transformer ratio, as shown in Fig. 2. Since the dc voltage in each $\mathrm{L}-\mathrm{CH}$ is $V_{d c} / 3$ the modulation index $m$ can be defined as:

$$
m=\frac{3 \cdot r \cdot \hat{V}_{\text {phase }}}{V_{d c}}
$$

Note that $m=1$ corresponds to the maximum ac side voltage that can be generated with conventional modulation. When the dc power matches the ac power, the power balance equation holds:

$$
V_{d c} \cdot I_{d c}=\frac{3}{2} \hat{V}_{\text {phase }} \cdot \hat{I}_{\text {phase }} \cos (\varphi)
$$

and then the relation between the dc and the ac current assumes the following expression,

$$
I_{d c}=\frac{1}{2} m \frac{\hat{I}_{\text {phase }}}{r} \cos (\varphi)
$$

As for the MMC, control of the dc and ac sides can be completely decoupled. The decoupling in the SCC is evident, since the sum of the dc voltage components of each $\mathrm{L}-\mathrm{CH}$ controls the dc side power transfer while the ac components control the ac side power.

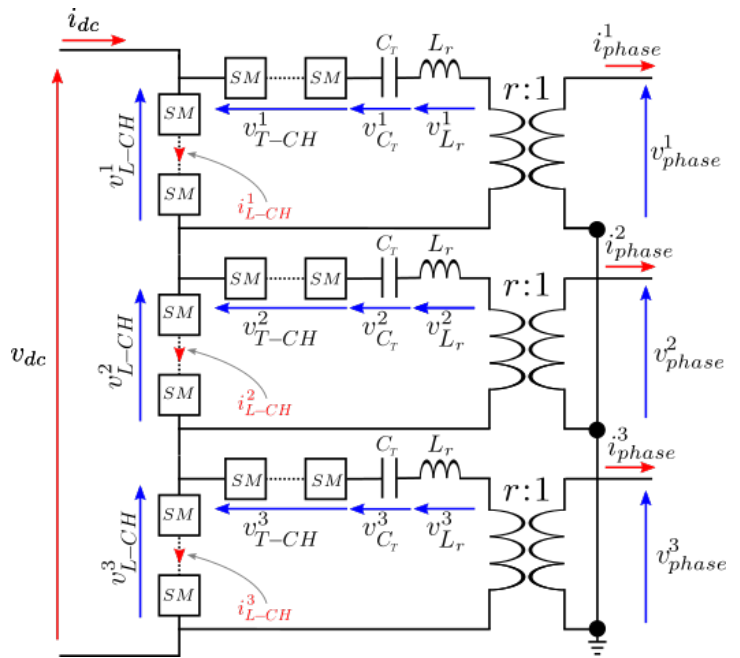

Fig. 2: Generalised representation of the SCC with inductors, capacitors and chain-links in the T-branches. Note that the ac line inductance is not shown and is assumed to be incorporated in the leakage inductance of the transformer in each phase.
The number of required SMs in each L-CH can be obtained using the eq. (1),

$$
N_{S M_{L-C H}}=\left\lceil\frac{\left|\hat{v}_{L-C H}^{i}\right|}{V_{S M}}\right\rceil
$$

where $\hat{v}_{L-C H}^{i}$ is the maximum voltage produced by each $\mathrm{L}-\mathrm{CH}$ and $V_{S M}$ is the nominal SM voltage. Eq. (6) can be rearranged and expressed as a function of the ac grid voltage. To do so, some assumptions are necessary. Firstly, it is assumed that there is no voltage generated across the Tbranches at the line frequency. To further simplify the analysis, the transformers are considered to be ideal and the voltage drop across the leakage inductance is neglected. In order to adjust the voltage levels during the normal converter operation, regulation margins must be guaranteed on both the $\mathrm{dc}$ and ac voltages. By defining the dc regulation margin $R M_{d c}$, the dc link voltage range can be expressed as:

$$
V_{d c}=\left(1 \pm R M_{d c}\right) V_{d c, N}
$$

where $V_{d c, N}$ is the dc link nominal voltage.

A similar expression can also be derived for the ac voltages,

$$
\hat{V}_{\text {phase }}=\left(1 \pm R M_{a c}\right) \cdot r \cdot \hat{V}_{\text {phase }, N}
$$

where $R M_{a c}$ is now the ac voltage regulation margin and $\hat{V}_{\text {phase }, N}$ is the nominal ac phase voltage. To produce the desired L-CH voltage with half-bridges, the ac voltage must always be smaller then the minimum dc voltage produced by each L-CH,

$$
\left(1-R M_{d c}\right) \frac{V_{d c, N}}{3} \geq\left(1+R M_{a c}\right) \cdot r \cdot \hat{V}_{\text {phase }, N}
$$

For given nominal ac and dc system voltages, and having fixed the regulation margins, eq. (9) can be used to identify the transformer turns ratio $r$. In addition to $R M_{d c}$ and $R M_{a c}$, a redundancy factor $k_{r}$ should be included. Taking into account the constraint (9), (6) can be used to determine the $N_{S M}$ in each L-CH, having fixed the design factors $\left(R M_{d c}, R M_{a c}\right.$ and $k_{r}$ ), the transformer turns ratio and the nominal $V_{S M}$. The resulting expression to determine the number of SM of the $\mathrm{L}-\mathrm{CH}\left(N_{S M_{L-C H}}\right)$ is given in the Appendix.

\section{B. T-branch options}

As highlighted earlier, the series connection of L-CHs on the dc side provides no path to eliminate the dc component (offset) from the ac side. Consequently, this must be removed by adding the T-branches. An important design aspect is the choice of the most convenient topology for the T-branches. Additionally, the T-branch can be designed to cooperate with L-CHs in dealing with unbalanced and fault conditions in the grid. Intuitively, it may be convenient to use the additional degree of freedom on the T-branch to guarantee, throughout the converter operating range, the constraint of a ripple free dc side,

$$
\sum_{i=1}^{3} v_{L-C H}^{i}(t)=V_{d c}
$$


If eq. (10) is not respected, ac components may appear on the dc side leading to deteriorated operation of the converter.

The minimum circuit configuration to block the dc offset on the ac side is a series capacitor. However, the dc blocking function should be performed with:

1) minimum size of the dc blocking circuit;

2) minimum impact on the ac voltage generation capability. These two objectives conflict, since with just a capacitor in the T-branch, it alone must be sized to guarantee negligible ac voltage drop. This leads to high values of capacitance and makes the solution impractical.

1) Passive T-branch: The limitations of a purely capacitive T-branch can be overcome by replacing the blocking capacitor with an LC (inductor $L_{r}$ and blocking capacitor $C_{T}$ ) series resonant tank, resonating at the line frequency. Doing so, the size of the capacitor can be reduced, and the ac voltage drop across the capacitor will be eliminated by the antiphase voltage across the resonant inductor. Note that this resonating inductor is seperate from the ac line inductance. The total impedance as seen from the grid is still inductive, assuming that the transformers are designed for a specific value of leakage inductance [23]. This solution is denoted as the Passive Tbranch SCC.

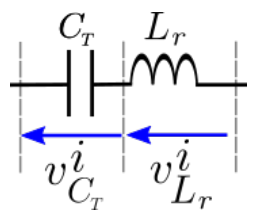

Fig. 3: Passive T-branch. The series connected inductor is selected to create a resonance tank, with the capacitor, at the line frequency.

Although dc offset elimination is achieved, an additional passive component is required (the inductor) and furthermore no "active" control of the T-branch can be achieved. Whilst it introduces a useful concept, the lack of flexibility in this solution is a serious limitation and we do not consider it in any further detail here.

2) Hybrid T-branch: The scheme proposed in Fig. 2 uses a combination of active components (chain-link of SMs - T$\mathrm{CH}$ ) and passive elements in the T-branches and will be referred to as a Hybrid T-branch SCC. In the Hybrid T-branch

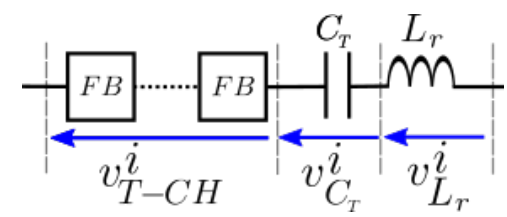

Fig. 4: Hybrid transverse T-branch, obtained with the series connection of a transverse full-bridge chain-link (T-CH), a capacitor $C_{T}$ and an inductor $L_{r}$.

in Fig. 4, the passive components are generally not chosen to resonate at the grid frequency. For this reason, the T-CH SMs are full-bridges, since, during normal operation the T-CH must produce an ac voltage able to cancel out the residual ac voltage drop across the passive components. This ac voltage is in quadrature with the line current and, neglecting loss, energy balance is naturally achieved in the T-CH SMs. In this sense, assuming $L_{r}<C_{T}$, the T-CH can be seen as an equivalent inductive reactance that makes the T-branch operate as a virtual resonant circuit where part of the inductance is passive and part is electronically emulated by the T-CH. The voltage produced by the $i^{t h} \mathrm{~T}-\mathrm{CH}$ can be generally expressed as:

$$
v_{T-C H}^{i}(t)=\omega_{g} \cdot L_{e q} \cdot \frac{1}{r} \hat{I}_{p h a s e}^{i} \sin \left(\omega_{g} \cdot t+\frac{\pi}{2}-\varphi-\frac{i-1}{3} \pi\right)
$$

where the equivalent inductance emulated by the $\mathrm{T}-\mathrm{CH}$ in order to achieve resonance at $\omega_{g}$ is:

$$
L_{e q}=\frac{1}{\omega_{g}^{2} \cdot C_{T}}-L_{r}
$$

As a result, the ac voltage drop at the grid frequency across the T-branch is zero. The passive resonant inductor cooperates with the $\mathrm{T}-\mathrm{CH}$ in eliminating the ac voltage drop across the blocking capacitor $C_{T}$. However, the retention of $L_{r}$ in the circuit could significantly impact the volume occupied by the converter. For this reason, the Hybrid T-branch may be reduced to a T-CH and a blocking capacitor $C_{T}$, with the T$\mathrm{CH}$ emulating all the required inductance (Fig.5).

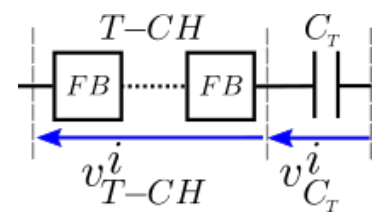

Fig. 5: Hybrid T-branch with a capacitor $C_{T}$ and a transverse full-bridge (FB) chain-link T-CH.

The equivalent inductance emulated by the $\mathrm{T}-\mathrm{CHs}$ is then,

$$
L_{e q}=\frac{1}{\omega_{g}^{2} \cdot C_{T}}
$$

It is now necessary to determine the value of $C_{T}$. This could be driven by an economic optimisation of the number of SMs in the T-CHs and the size of the blocking capacitors $C_{T}$. However, at this stage the capacitor is simply selected in order to limit the ac voltage drop, due to the nominal ac current on the converter side, to a value equal to half of the ac voltage produced by each L-CH,

$$
X_{C_{T}} \cdot \frac{I_{\text {phase }, N}}{r}=r \cdot \frac{V_{\text {phase }, N}}{2}
$$

where $I_{\text {phase, } N}$ and $V_{\text {phase, } N}$ are, respectively, the nominal values of the current and phase voltage on the grid side. Using eq. (14) the required capacitance can be obtained,

$$
C_{T}=2 \cdot \frac{I_{\text {phase }, N}}{r \cdot \omega_{g} \cdot V_{\text {phase }, N}}=\frac{1}{\pi f_{g}} \frac{S}{\left(r V_{L L}\right)^{2}}
$$

where $\mathrm{S}$ is the rated apparent power and $V_{L L}$ is the grid line to line voltage. The number of T-CH SMs can be determined according to voltage drop on $C_{T}$ that needs to be cancelled (half the converter side ac voltage in this case). However, extra voltage capability could be useful to cover all the contingencies [24], [25]. For this reason, it has been assumed that each $\mathrm{T}-\mathrm{CH}$ must be able to produce $3 / 5^{t h}$ of the nominal phase voltage,

$$
\max \left(V_{T-C H}\right)=\frac{3}{5} \cdot r \cdot \hat{V}_{\text {phase }, N}
$$


and the number of required SMs can be determined according to (39) given in the Appendix.

3) Active T-branch: Finally, Fig. 6 illustrates another possible arrangement of the T-branch that is realised only using half-bridge SMs controlled to produce 1/3rd of the dc voltage:

$$
V_{T-C H}^{i}=\frac{V_{d c}}{3}
$$

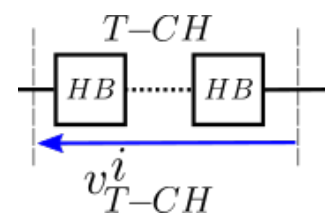

Fig. 6: Active transverse T-branch. The connection features only a transverse half-bridge $(\mathrm{HB})$ chain-link $(\mathrm{T}-\mathrm{CH})$.

This is the most intuitive solution, as the dc offset is removed by placing an equivalent controllable voltage source such that the polarity opposes the corresponding dc component of the $v_{L-C H}^{i}$. As derived for the Hybrid T-CH and the L-CH an expression to identify the required minimum number of SMs in the active T-CHs $N_{S M_{T-C H}}^{\text {active }}$ can be obtained and is given in the Appendix.

\section{Selection of the optimal converter topology}

In this subsection some considerations are presented to choose the most suitable solution between the Hybrid and the Active SCC. As highlighted, this main aim of this new topology is to reduce the number of SMs in the converter, as a higher number increases the control system complexity and, in turn, the overall converter complexity. The main operating differences between the options have been presented and now a comparison in terms of the ratings of the T-CHs is given.

1) Current rating: In both solutions the current flowing in the two T-CH is the same, and the semiconductors will have the same current ratings.

2) Number of SMs: The number of required SMs can be determined via eq. (39) and (40) given in the Appendix, and the relative requirement between the two options can be obtained as,

$$
\frac{N_{S M_{T-C H}}^{\text {Hybrid }}}{N_{S M_{T-C H}}^{\text {Active }}} \approx 0.6
$$

For simplicity the $R M_{d c}$ and $R M_{a c}$ have been assumed equal to 1 . Note that if an ac regulation margin $R M_{a c}$ less than unity is used (it is sensible to assume so as otherwise no ac regulation margin would be available) the relative SM requirement of (18) decreases and becomes even more favourable to the Hybrid SCC.

From (18) it can be concluded that the Active SCC requires at least $40 \%$ more SMs than the Hybrid SCC. However, the Hybrid SCC requires full-bridge modules whereas the Active SCC requires only half bridges. As consequence the semiconductor count for the two options is similar.
3) Energy storage: To provide a basic evaluation of the different energy storage requirements, the instantaneous power $p_{C H}(t)$ absorbed by each chain-link can be integrated over time (for the power balance condition with zero average power exchanged by the chain-links) and the energy variation $\Delta e(t)$ can be derived,

$$
\Delta e(t)=\int p_{C H}(t) d t=v_{C H}(t) \cdot i_{C H}(t) d t
$$

Also, assuming perfect energy sharing among the SMs within a chain-link, the time dependant energy stored in the chain-link can be described by

$$
E_{C H}(t)=\frac{1}{2} C_{S M} N_{S M} v_{S M}^{2}(t)
$$

The instantaneous voltage in each SM $v_{S M}(t)$ can be decomposed into a dc component $V_{S M}$ and an ac component $\Delta v_{S M}(t)$. By assuming power balance conditions and small ripple in $v_{S M}(t)$, the expression of the energy fluctuation as a function of the ac component of the SM voltage becomes:

$$
\Delta e(t) \approx C_{S M} \cdot N_{S M} \cdot V_{S M} \cdot \Delta v_{S M}(t)
$$

Knowing the expression of the energy fluctuation, the peak to peak ac voltage component can be limited by selecting an appropriate value of $C_{S M}$ according to

$$
C_{S M} \geq \frac{\Delta e_{p p}}{N_{S M} \cdot \delta_{v_{c, p p}} \cdot V_{S M}^{2}}
$$

where $\delta_{v_{c, p p}}$ is the per unit peak-to-peak voltage fluctuation, normalised with respect to the nominal SM voltage $V_{S M}$. The minimum energy storage in each chain-link can be calculated as:

$$
E_{\text {min }}=\frac{\Delta e_{p p}}{2 \cdot \delta_{v_{c, p p}}}[J]
$$

Also, the minimum specific energy required $H$ [26] to guarantee the per unit peak-to-peak voltage fluctuation $\delta_{v_{c, p p}}$, can be written as:

$$
H_{\text {min }}=\frac{\Delta e_{p p}}{2 \cdot \delta_{v_{c, p p}} \cdot S}\left[\frac{J}{W}=s\right]
$$

In the literature $\mathrm{H}$ is generally expressed in $k J / M W$ or $m s$. Eq. (23) and (24) can be used for a comparison of the two T-branch solutions. It is first necessary to evaluate the energy fluctuation in the individual T-CHs. During normal operation, the amplitude of the voltage produced by the Hybrid $\mathrm{T}-\mathrm{CH}$ can be approximated by,

$$
\hat{V}_{T-C H}^{i}=\omega_{g} \cdot L_{e q} \cdot \frac{1}{r} \hat{I}_{\text {phase }}^{i} \approx r \cdot \frac{\hat{V}_{\text {phase }}^{i}}{2} \approx \frac{V_{d c}}{6}
$$

To explain the expression above some remarks are required. The T-CH is controlled, as already discussed, to "resonate" with the blocking capacitor $C_{T}$ and at nominal operating conditions it produces an amplitude that is half of the amplitude of the nominal ac voltage, that in turn is roughly half the dc voltage produced by each $\mathrm{L}-\mathrm{CH}$. The peak to peak energy fluctuation can be estimated by,

$$
\Delta e_{p p}^{H y}=2 \frac{V_{d c} \hat{I}_{\text {phase }}^{i}}{24 \omega_{g} r} \approx \frac{S}{6 \omega_{g}}
$$


The energy fluctuation in the Active $\mathrm{T}-\mathrm{CH}$ of the i-th phase can be evaluated as

$$
\Delta e_{p p}^{A c t}=2 \frac{V_{d c} \hat{I}_{p h a s e}^{i}}{3 \omega_{g} r} \approx \frac{4}{3} \frac{S}{\omega_{g}}
$$

The relative energy storage requirement can now be evaluated as:

$$
\frac{\Delta e_{p p}^{A c t}}{\Delta e_{p p}^{H y}}=8
$$

The energy storage in the Active $\mathrm{T}-\mathrm{CH}$ is eight times greater than the energy storage in the Hybrid T-CH solution. This result comes about because of the different energy fluctuation ripple and the different amplitude of the voltage produced by the two solutions. The Hybrid chain-link produces approximately half the voltage of the Active $\mathrm{T}-\mathrm{CH}$ and the ripple frequency is double that of the Active T-CH.

The smaller energy storage requirement of the Hybrid solution makes it the most attractive option for the T-branch configuration. Consequently the hybrid option is only one taken forward for further study and for simplicity we remove the hybrid designation and just refer to it as the SCC.

\section{Discussion on energy storage and number of SMs of the selected topology}

Using eq. (1) and eq. (2) it is possible to determine the total energy fluctuation in the L-CH SMs. It is easily shown that the total energy fluctuation is equal to the energy fluctuation of an equivalent MMC. Consequently, the energy storage in the L-CHs is approximately the same as an equivalent MMC. To evaluate the additional storage required by the T-CHs eq. (26) can be used. As $V_{d c} / 3 \approx r \cdot V_{\text {phase }}^{i}$, eq. (26) can be rewritten as:

$$
\Delta e_{p p}^{H y} \approx 2 \frac{V_{p h a s e}^{i} \hat{I}_{\text {phase }}^{i}}{4 \omega_{g}}
$$

The specific energy storage for the T-CHs can be written in the form of,

$$
H^{H y}=\frac{\Delta e_{p p}^{H y}}{S} \approx 2 \cdot \frac{S}{4 \omega_{g}} \frac{1}{S}=\frac{1}{2 \pi f_{g}}
$$

For example, a system with a line frequency $f_{g}=50 \mathrm{~Hz}$ requires and additional specific energy of approximately 3 $\mathrm{kJ} / \mathrm{MVA}$. Hence the additional energy stored in the T-CHs is not significant since it is, for example, less than $10 \%$ of the typical total energy storage in an MMC [27].

The number of SMs required by an MMC can be identified according to the system voltage levels and the available semiconductor devices. However, a simplified comparative analysis to identify the relative SM requirement between two modular multilevel converters can be determined by approximating the available voltage in each chain-link of SMs of the complete converter in terms of the dc voltage. Thus, the total requirement for the $\mathrm{SCC}$ is $\approx 2 V_{d c}+3 \cdot V_{d c} / 6$ while for an equivalent $\mathrm{MMC}$ is $\approx 6 V_{d c}$. This means that an SCC requires approximately the same energy storage as an equivalent MMC and the number of SMs required is reduced by $\approx 60 \%$. This result can be seen also from a different perspective: the SCC has the potential to compact the energy storage of an MMC into a converter that requires only $40 \%$ of the number of SMs of an MMC.

\section{HVDC SIMULATION AND COMPARISON}

In order to prove the viability of the SCC topology introduced in this paper, the results of a full switching simulation are presented in this section. The voltage and power ratings of a scaled down industrial HVDC (MMC) demonstrator [28] have been used as reference for this study. Fig. 7 shows the simplified schematic of the SCC HVDC system considered.

The converter operates as an interface between a medium voltage ac grid and a dc system. For simplicity the dc system is replaced by a resistive load $R_{L O A D}$. The parameters of the two systems are summarised in TABLE I.

TABLE I: HVDC scaled down system parameters

\begin{tabular}{lll}
\hline \hline Symbol & \multicolumn{1}{c}{ Quantity } & Value \\
\hline$V_{d c}$ & dc voltage & $20 \mathrm{kV}$ \\
$V_{L L}$ & line to line voltage & $11 \mathrm{kV}$ \\
$f_{g}$ & line frequency & $50 \mathrm{~Hz}$ \\
$P$ & Active power & $20 \mathrm{MW}$ \\
$Q$ & Reactive power & $8.5 \mathrm{MVAr}$ \\
\hline \hline
\end{tabular}

On the ac side each converter phase is connected to the corresponding grid phase via a single phase transformer as shown in Fig. 7. It is very important to note that $L_{s}$ shown in Fig. 7 is not the same as $L_{r}$ shown previosly in Fig. 4. The function of $L_{r}$ is taken up entirely by the chain-links as explained previously while $L_{s}$ represents the ac line interface reactance required by any HVDC converter to allow for power control etc. It includes the transformer leakage reactance and is common to both the SCC and MMC implementations and

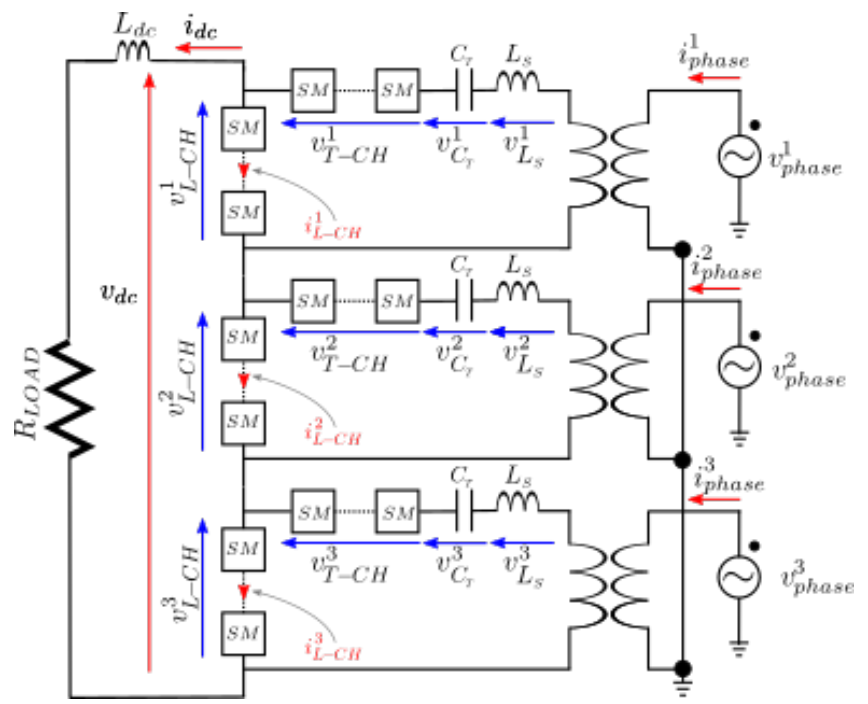

Fig. 7: Schematic of the simplified SCC HVDC system for comparison study. 
it is not therefore included in the comparison tables. A value of $18 \%$ is used according to the value reported in [28]. The transformer turns ratio is identified by using (9) having fixed the $\mathrm{dc}$ and an ac voltage regulation margin $\left(R M_{d c}, R M_{a c}\right)$ both equal to 0.05 ( $\pm 5 \%$ voltage regulation). On the dc side the system is modelled as a resistive load of $20 \Omega$ which is connected to the converter via an inductor $L_{d c}=22 \mathrm{mH}$.

TABLE II: SCC parameters

\begin{tabular}{lll}
\hline \hline \multicolumn{1}{c}{ Symbol } & \multicolumn{1}{c}{ Quantity } & \multicolumn{1}{c}{ Value } \\
\hline & & \\
$C_{S M_{L-C H}}$ & L-CH SM capacitance & $19 \mathrm{mF}$ \\
$C_{S M_{T-C H}}$ & T-CH SM capacitance & $12 \mathrm{mF}$ \\
$C_{T}$ & Transverse capacitance & $2.3 \mathrm{mF}$ \\
$N_{S M_{L-C H}}$ & Number of L-CH SM per phase & 11 \\
$N_{S M_{T-C H}}$ & Number of T-CH SM per phase & 5 \\
$V_{S M}$ & SM operating voltage & $1.5 \mathrm{kV}$ \\
$f_{P W M}$ & PWM frequency & $500 \mathrm{~Hz}$ \\
$f_{\text {sort }}$ & Sorting frequency & $500 \mathrm{~Hz}$ \\
\hline \hline
\end{tabular}

The capacitance values of the sub-modules have been selected according to (22) in order to limit the voltage oscillation to around $\pm 10 \%$ of the average value (i.e. $20 \%$ peak to peak voltage fluctuation $\delta_{v_{c, p p}}$ ), [29]. The number of SMs for the L$\mathrm{CHs}$ and T-CHs are calculated respectively according to (38) and (39) assuming $10 \%\left(k_{r}=0.1\right)$ SMs redundancy. The SM operating voltage is $1.5 \mathrm{kV}$ [28] and IGBT modules with $3.3 \mathrm{kV}$ voltage ratings are considered. The full-load losses for this basic design assuming typical $3.3 \mathrm{kV}$ devices has been calculated at $1.3 \%$ - a figure that could be further optimised by examining trade-offs between losses and fault handling capabilities [24].

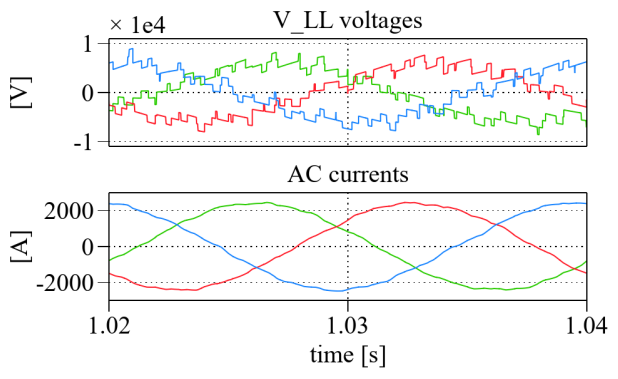

Fig. 8: Line to line voltages and ac currents on the converter side of the transformer.

In Fig. 8 the waveforms of the ac voltages and currents are shown. This figure shows the ac quantities measured on the converter side of the transformer. The ac current has THD of $2.5 \%$.

On the dc side the voltage $V_{d c}$ of the converter is the sum of the three voltages produced by the L-CHs according to (10). The dc voltage shows a ripple at the switching frequency given by the instantaneous sum of the three L-CH SMs that are pulse width modulated (one for each L-CH). This voltage oscillation has a range of $\pm 2 \cdot V_{S M}$.

The current flowing in each $\mathrm{L}-\mathrm{CH}$ is given by the sum of the dc current and the ac current of the corresponding phase as shown in Fig. 10.

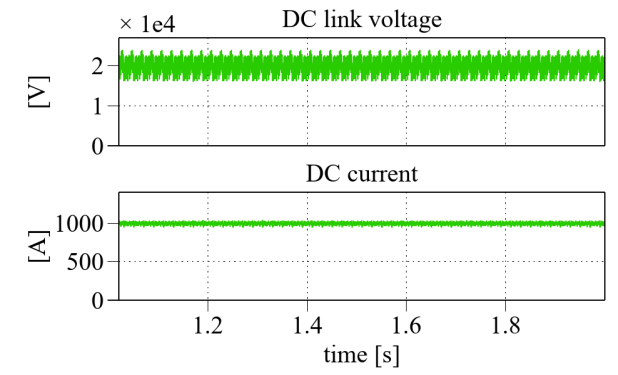

Fig. 9: The top waveform shows the dc side voltage produced by the converter. The bottom waveform shows the dc current flowing into the dc load

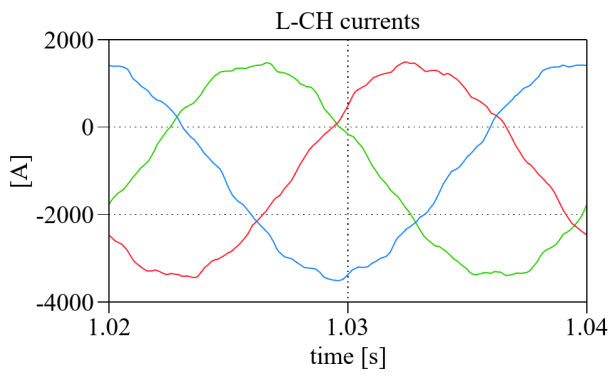

Fig. 10: Waveforms of L-CH currents.

The characteristic voltage waveforms of the transverse branches are shown in Fig. 11. This shows the voltages on the transverse capacitors $C_{T}$ and the voltages produced by the T-CHs. As expected each $V_{C_{T}}$ voltage has a dc component

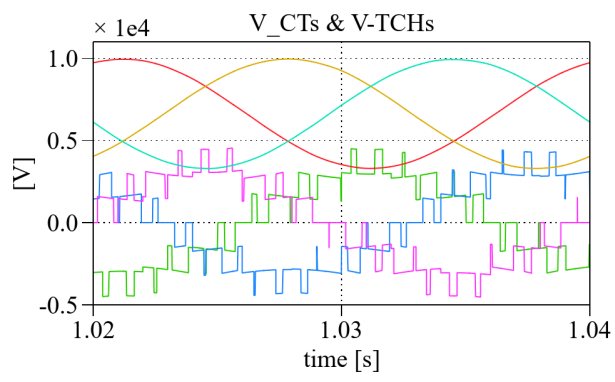

Fig. 11: Converter SM voltages

approximately equal to $V_{d c} / 3$ and an ac component equal to the capacitive voltage drop. The T-CHs are modulated to produce a voltage that cancels this capacitive voltage drop.

The SM nominal voltages $\left(V_{S M}\right)$ have been chosen to be $1.5 \mathrm{kV}$ and the instantaneous waveforms are shown in Fig. 12. As expected, the fundamental period of the voltage ripple is $20 \mathrm{~ms}$ for the L-CH SMs (the same as in an MMC) whereas it is $10 \mathrm{~ms}$ for the T-CH SMs.

\section{MMC comparison}

The SCC design used to simulate the scaled down industrial HVDC system is used in this section for a comparison with an equivalent MMC. The MMC is assumed to be connected between the same dc system and ac grid. Equivalent design methods, already introduced for the SCC have been used to determine the parameters of the MMC converter. In order to choose the transformer ratio used to interface the MMC with 


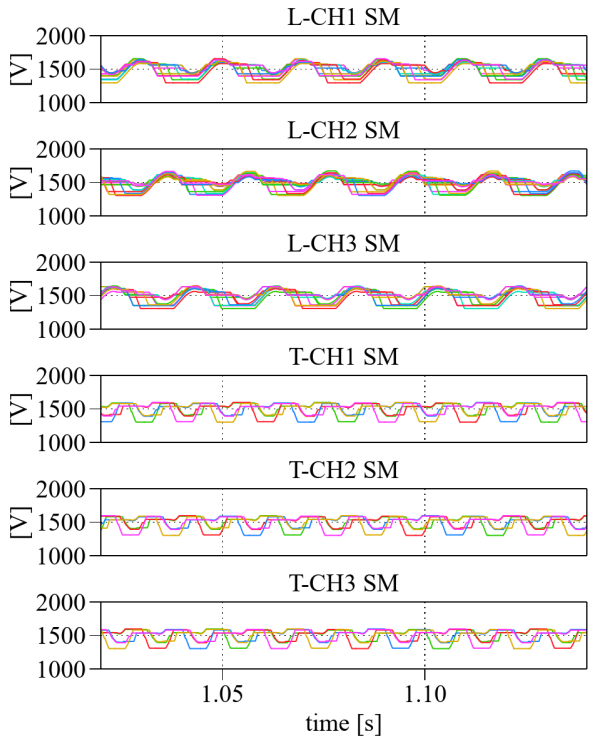

Fig. 12: Converter SM voltages

the ac grid the same ac and dc regulation margins introduced for the SCC have been used. The average SM voltage is $V_{S M}=1.5 \mathrm{kV}$, the value of the SM capacitance is selected to limit the peak to peak voltage oscillation to $20 \%$ of the average SM voltage and the number of SMs have been determined considering the same redundancy adopted for the SCC (i.e. $10 \%)$.

TABLE III: Comparison.

$* \mathrm{~L}-\mathrm{CH}, * * T-\mathrm{CH}$

\begin{tabular}{lll}
\hline \multicolumn{1}{c}{ Quantity } & MMC & \multicolumn{1}{c}{$\mathrm{SCC}$} \\
\hline$r$ & 1.05 & 0.7 \\
$N$ of chain-links & 6 & 6 \\
$N_{S M}$ per chain-link & 16 & $11^{*}, 5^{* *}$ \\
$N_{S M}$ per converter & 96 & $48=33^{*}+15^{* *}$ \\
$V_{S M}$ & $1.5 \mathrm{kV}$ & $1.5 \mathrm{kV}$ \\
$I_{c h(R M S)}$ & $0.7 \mathrm{kA}$ & $1.9^{*} \mathrm{kA}, 1.6^{* *} \mathrm{kA}$ \\
$I_{d c}$ & $1 \mathrm{kA}$ & $1 \mathrm{kA}$ \\
$C_{S M}$ & $7.5 \mathrm{mF}$ & $19^{*} \mathrm{mF}, 12^{* *} \mathrm{mF}$ \\
$H$ & $36.3 \mathrm{~ms}$ & $32.5^{*} \mathrm{~ms}, 9.3^{* *} \mathrm{~ms}$ \\
$C_{T}$ & - & $2.3 \mathrm{mF}$ \\
$H_{C_{T}}$ & - & $7 \mathrm{~ms}$ \\
$L_{\text {arm }}$ & $4.7 \mathrm{mH}$ & - \\
$N$ of arm inductors & 6 & - \\
\hline \hline
\end{tabular}

The results of the comparison are summarised in TABLE III. The main advantage of the topology is the reduction of number of SMs, indeed the SCC requires 50\% fewer SMs than an MMC. This factor significantly impacts on the required control apparatus and auxiliary elements to be installed. Also the number of semiconductors is reduced. In addition the inherent concentration of the energy storage, although a greater specific value is needed ( $15 \%$ more), could help to reduce the occupied volume since the energy is stored in less SMs. Three transverse capacitors $C_{T}$ and one dc side inductor $L_{d c}$ are required, reducing the number of passive elements from 6 (one arm inductor for each MMC arm) to 4. It can be seen from the table that the major drawback of the converter is the high current circulating in the semiconductors. This preliminary comparison shows as the SCC has the potential to reduce those factors that significantly impact the converter footprint, namely the number of SMs and the number of passive elements.

\section{Control strategy}

The main objective of this section is to develop the control scheme for the SCC. This analysis focuses on the control of the small scale laboratory prototype of the SCC that has been designed and built to validate the converter concept. Only the control loops that are strictly necessary for the operation of the laboratory demonstrator are presented, a more detailed analysis of the converter dynamics will be the object of future work. The basic schematic of the laboratory prototype set-up is the same as that used for the simulation study shown previously in Fig. 7

where it is worth noting that the inductors $L_{s}$ on the ac side are the combination of interconnection inductors with the leakage inductance of the transformer, and they are not used "to cancel" the blocking capacitor ac voltage drops. This task is fully carried out by the T-CHs. For simplicity, control of the voltage on the dc side is performed in open loop. The dc voltage is shared equally among the three L-CHs and constitutes the constant component of the $\mathrm{L}-\mathrm{CH}$ modulation signals. The power absorbed by the dc side is therefore defined by the resistive load. A slow outer total energy control generates the active components of the ac current references in order to meet the power balance, and these are tracked by an inner current controller. A reactive current component, $I_{R}^{\text {ref }}$, can be directly added to the ac current references.

\section{A. Current control}

Considering that the outer energy control loops will be designed to have dynamics at least an order of magnitude slower than the current loops, the T-CHs and the L-CHs can be considered as ideal controllable voltage sources. The current loops can then be analysed referring to a generic i-th phase, and the ac side equation can be derived from Fig. 7 as:

$$
\begin{aligned}
r \cdot v_{\text {phase }}^{i}(t) & =\frac{1}{C_{r}} \int i_{\text {phase }}^{i}(t) d t+L_{s} \frac{d i_{\text {phase }}^{i}(t)}{d t} \\
& +v_{L-C H}^{i}(t)-v_{T-C H}^{i}(t), i=1,2,3
\end{aligned}
$$

For simplicity, the full-bridge chain-links in the hybrid Tbranches discussed in Section II-B2, are controlled in openloop so that the generated ac voltages $v_{T-C H}^{i}(t)$ are "inductive" $50 \mathrm{~Hz}$ voltages that cancel the ripple across $C_{T}$ in each phase. The modulation signals for $v_{T-C H}^{i}(t)$ are derived from the ac current references and the value of the capacitors $C_{T}$. As a result, the voltages $v_{T-C H}^{i}(t)$ act as a $50 \mathrm{~Hz}$ disturbance for the small signal model of the current control. For this reason, the chain-link that controls the current of the $\mathrm{i}$-th phase is only the corresponding $v_{L-C H}^{i}$. In conclusion, 
the equivalent circuit of the ac side can be represented as in Fig. 13, where the disturbance introduced by the T-CH generator has been neglected for brevity.

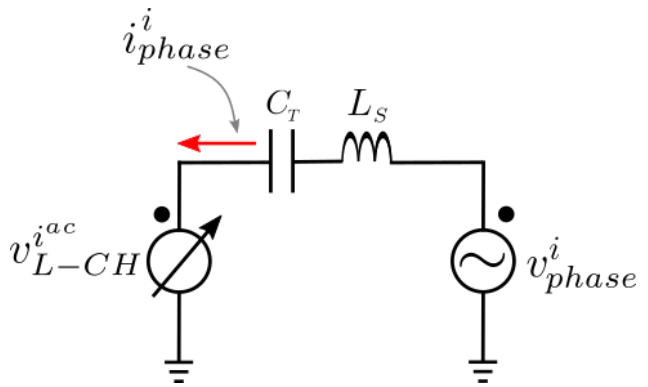

Fig. 13: Equivalent ac circuit of the i-th converter phase $(i=1,2,3)$.

The current control proposed in this paper is realised in the abc frame and three single phase PLLs based on quadrature signal generation and the Park transformation [30] are used for grid synchronisation. In order to track the alternating current reference a Proportional-Resonant (PR) controller, derived in [31], has been used for each phase as shown in Fig. 14.

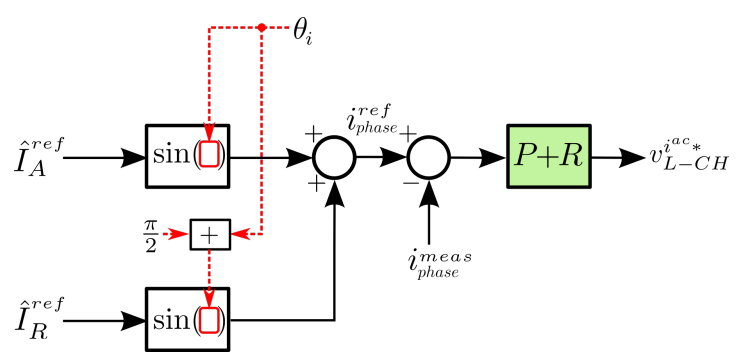

Fig. 14: Current control loops.

The output of the controller constitutes the ac modulation signal $v_{L-C H}^{i^{a c *}}$ of the corresponding L-CH modulator.

\section{B. Energy control}

The energy control should guarantee that the total energy stored in the converter is maintained at the reference value and that uniform energy distribution is achieved within each chainlink. These needs are met via a three level control structure that consists of: Total Energy Control (TEC), Inter-phase control (IPC) and Intra Chain-link Balancing (ICB).

The dynamics of the average voltage in each chain-link are approximated by the s-domain transfer function that can be obtained via linearising the non-linear differential equation that describes the system,

$$
G_{P}=\frac{V_{C_{e q}}(s)}{P(s)}=\frac{1}{s V_{S M} C_{S M}}
$$

This transfer function describes the relationship between the input power $P(s)$ and the output voltage $V_{C_{e q}}(s)$ of a generic chain-link of SMs about the quiescent working point.

1) TEC control: The TEC in Fig. 15 ensures that the total energy stored in converter SMs is maintained at the desired level. To do so, this control acts simultaneously on both the L-CHs and the T-CHs total energies. The reference voltage $V_{C_{e q} \sum}^{r e f}=\left(3 \cdot N_{S M_{L-C H}}+3 \cdot N_{S M_{T-C H}}\right) \cdot V_{S M}^{r e f}$ is compared with feedback of the total voltage "available" in the converter
$V_{C_{e q} \sum \text {, }}^{\text {meas }}$, i.e. the sum of all the SM voltages, and the error is processed by a Proportional-Integral (PI) controller that defines the peak of the active component of the grid current reference, thus defining the ac active power. However, a small part of this power must be "intercepted" by the T-CHs in order to maintain the local energy balance by compensating for semiconductor loss. To do so a resistive voltage component is added to the $v_{T-C H}^{i}$ of (11) via a controllable angle $\delta^{i}$,

$$
v_{T-C H}^{i}=-\omega_{g} \cdot L_{e q} \cdot \frac{1}{r} \hat{I}_{r e f} \sin \left(\omega_{g} \cdot t+\frac{\pi}{2}-\varphi-\frac{i-1}{3} \pi-\delta^{i}\right)
$$

The measured "available voltage" in the T-CH of the ith phase $V_{C_{e q}\left(T-C H^{i}\right)}^{\text {meas }}=\sum_{j=1}^{N_{S M_{T-C H}}} v_{T-C H^{i}}^{j}$ is subtracted from the reference voltage $N_{S M_{T-C H}} V_{S M}$ and then the output of a PI controller defines the controllable angle $\delta^{i}$ of the corresponding T-CH. The modulation signal of the corresponding $\mathrm{T}-\mathrm{CH}$ is defined by a voltage function according to (33).

2) IPC control: During the converter operation, mismatches between the energy stored in the different phases can arise. It is then necessary to add another level of energy control to equalise the energy storage. This control acts on the net power exchange between different converter phases. However, it does not modify the power reference generated by the TEC, but it is designed to distribute the power reference differently between the converter phases to reach energy equalisation. It is worth noting that only the differential energy of two pairs of phases are required since the third is a combination of the other two. The implementation of this additional level of control is shown in Fig. 16.

Considering that the dc power is imposed by the load and $V_{d c}$, by adjusting the contribution of each $\mathrm{L}-\mathrm{CH}$ to the $\mathrm{dc}$ voltage it is possible to modify the net power exchanged by the chain-link. Each L-CH dc component is modified via the corresponding $\Delta V_{d c}^{*}$. It is important to remark that the IPC does not affect the power exchanged on the dc side and the sum of the three dc components $\Delta V_{d c_{1,2,3}}^{*}$ is always zero. The parameters of the controllers are selected in order to obtain a dynamic response slower than the TEC loops to guarantee decoupling.

3) Modulation and ICB control: The control loops generate the modulation signals for the converter chain-links, however they are generated assuming ripple-free SM voltages. If no additional action is taken, the interaction between the modulation signals and the SM voltage ripple will introduce chainlink voltage components at multiples of the line frequency. To avoid this, the modulation index $\operatorname{Mod}(t)$ is compensated with the available voltage in the corresponding chain-link $V_{C_{e q}}(t)$ according to,

$$
\operatorname{Mod}^{c}(t)=\frac{\operatorname{Mod}(t)}{V_{C_{e q}}(t)} \cdot N_{S M} \cdot V_{S M}
$$

$\operatorname{Mod}^{c}(t)$ is the compensated modulation signal of a generic chain-link of SMs. The modulation technique used to produce the gate signals for the SM switches is based on the Phase Disposition pulse width modulation (PD-PWM) technique.

The ICB is used to equalise the SM voltages with a canonical sorting procedure [32], [33] and the algorithm implemented to sort the SMs is the so-called "bubble sort" - although it 


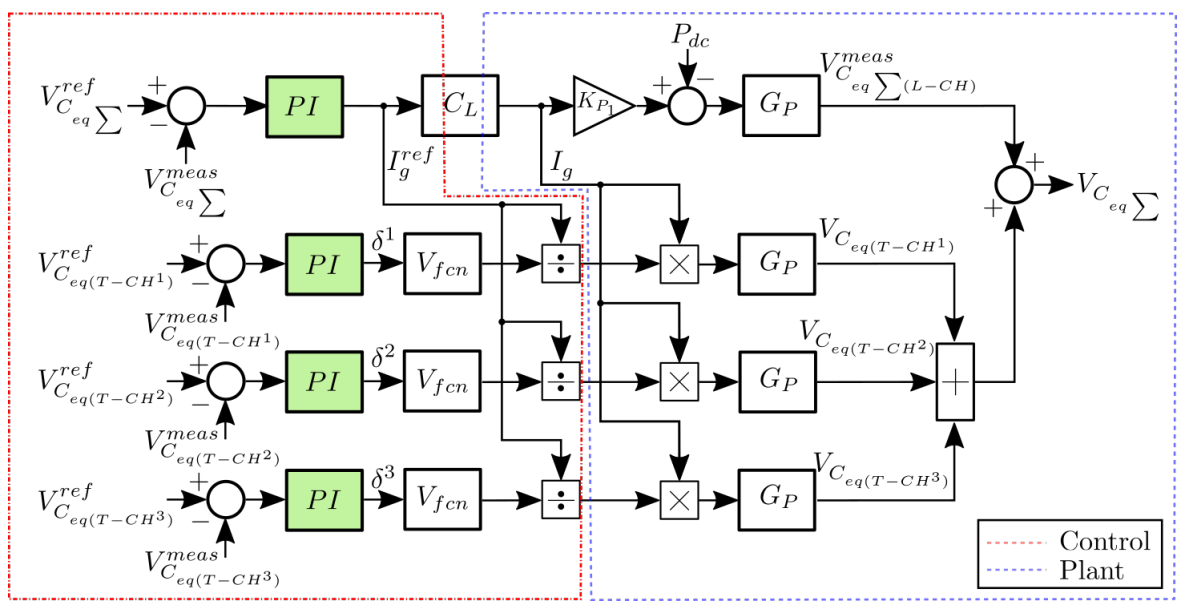

Fig. 15: TEC control loops. $K_{P_{1}}$ is the power constant and it is equal to the RMS value of the ac grid voltage. The TEC includes the PI that controls the voltage in the L-CHs (top PI) and the 3 PIs that control the voltage in the T-CHs. $C_{L}$ is the closed loop tranfer function of the current control. The voltage function $V_{f c n}$ is implemented according to (33)

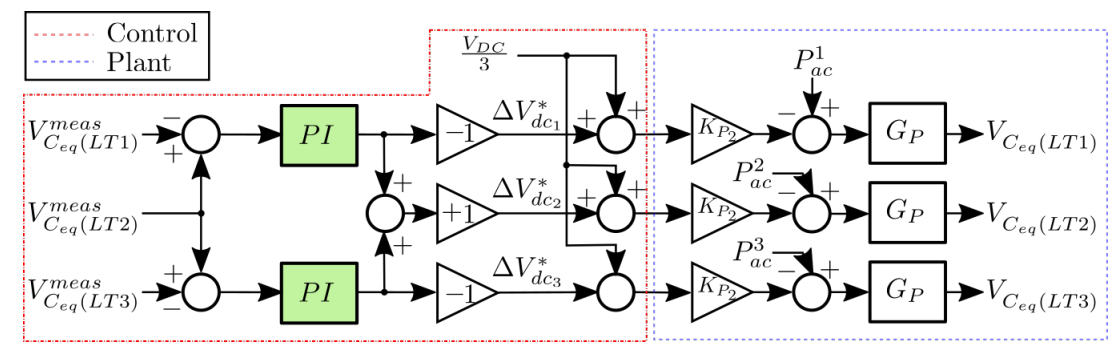

Fig. 16: IPC control loops. $K_{P_{2}}$ is the power constant, that in this control loop is the value of the dc current. $G_{P}$ is the transfer function between the voltage in the phase chain-links and the input power. The sign of the $\Delta V_{d c_{1,2,3}}^{*}$ depends on the direction of the power flow. $V_{C_{e}} q(L T i)$ is the available voltage in the $\mathrm{L}-\mathrm{CH}$ and the $\mathrm{T}-\mathrm{CH}$ of the ith phase

should be noted that any previously reported sorting method could be used. The sub-modulation signals will be sorted according to the sign of the current in order to equalise the voltage sharing within each chain-link.

\section{EXPERIMENTAL SETUP}

To validate the converter, a flexible modular multilevel prototype for laboratory purposes has been designed and built. The control system is based on a master-slave architecture designed to achieve high control flexibility.

\section{A. Laboratory prototype}

The converter has been designed for a nominal ac RMS line to line voltage of $400 \mathrm{~V}$ and a dc voltage of $450 \mathrm{~V}$. The total power rating is equal to $4.5 \mathrm{kVA}$ with a power factor (PF) of 0.8. It is worth noting that each $\mathrm{L}-\mathrm{CH}$ should be able to reproduce a third of the dc voltage plus the corresponding ac voltage on the converter side, as indicated in (35).

$$
V_{M A X}=\frac{V_{d c}}{3}+r \hat{V}_{\text {phase }}
$$

With this assumption, the maximum peak value of the ac voltage produced by the converter cannot exceed $150 \mathrm{~V}$ (when the converter is operated at $450 \mathrm{~V}$ on the dc side): as a consequence, the transformer turns-ratio has been selected to be equal to 240/90. This will leave, in nominal operating conditions, at least $10 \%$ ac voltage margin.

With the power rating defined, a sensible number of SMs should be selected to produce the multilevel waveforms output by the converter. Accordingly, 5 half-bridge SMs $\left(N_{S M_{L-C H}}\right)$ are used for each L-CH and the nominal voltage of each $\mathrm{L}-\mathrm{CH}$ $\mathrm{SM}$ is $60 \mathrm{~V}$ according to (36).

$$
V_{S M_{L-C H}}=\frac{V_{M A X}}{N_{S M_{L-C H}}} \simeq \frac{V_{d c}}{3} \frac{2}{N_{S M_{L-C H}}}
$$

To simplify the the design, the T-SMs have also been chosen with the same voltage rating and 3 full-bridge SMs have been adopted in each T-CH. The switching devices are Infineon IPB072N15N3 MOSFET, rated for $150 \mathrm{~V}$ and 100A. The capacitor in each $\mathrm{SM}$ is $5 \mathrm{mF}$, giving a equivalent $H$ constant [26] of $50 \mathrm{~ms}$. Two series connected resistors are connected across the terminals of the "SM floating dc link" for discharging and measurement purposes. It is worth noting that less energy storage ( $H=38 \mathrm{~ms})$ is actually required to achieve the $10 \%$ voltage ripple in all SM because the SM in the T-CHs could have a lower capacitance. However, the same capacitance has been used in all the SMs for ease of manufacturing. Each phase of the converter is realised by stacking 8 SMs (5 L-CH and $3 \mathrm{~T}-\mathrm{CH}$ ) and connecting them to the same backplane, which interfaces a local slave controller with the control and sensing signals in each SM. 


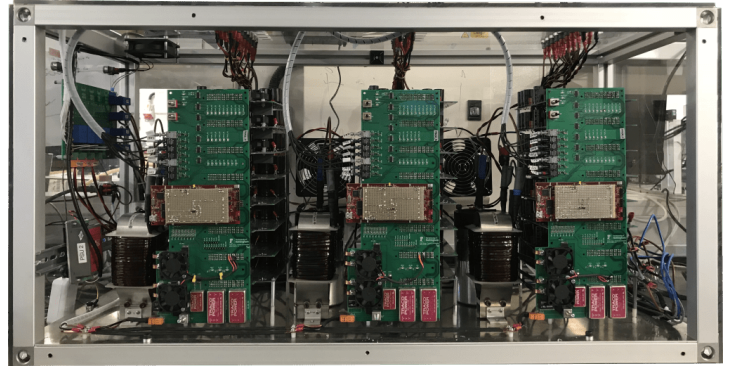

Fig. 17: Photograph of the experimental rig.

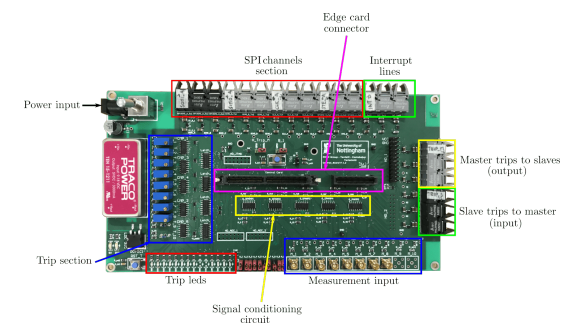

Fig. 18: Central controller docking board.

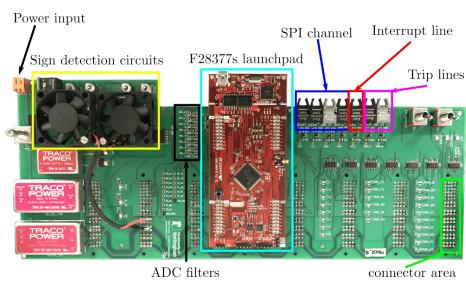

Fig. 19: Local control board.

\section{B. Control system}

In this application, the control system has been implemented using off-the-shelf micro-controller-units (MCUs). Monitoring all the SM voltages to ensure control of the energy stored in the SMs requires a number of ADCs that are not typically available in commercial MCUs. However, it is worth noting that SM voltage control is performed on the total voltage of each chain-link and not on the single SM. This allows a control based on a master-slave architecture to be used, where a local slave MCU monitors each SM in the chain-link and communicates to the master only the sum of the available voltages. In the SCC prototype, the central control board hosts a Texas Instruments F28379d controlCARD, acting as master (Fig. 18). The master is connected to the local controllers via optical channels and to a measurement board via coaxial cables. In each SM stack, a F28377s Launchpad sits on a local controller backplane (Fig. 19).

\section{EXPERIMENTAL RESULTS}

In order to initially validate the new topology and the associated control scheme implemented in the experimental rig (TABLE IV), full switching simulations have been performed in the MATLAB/Simulink environment in conjunction with the PLECS blockset. However, due to space the experimental results are presented in preference in the following paragraphs. However, to illustrate the match between the simulation study and the experimental tests the dynamic responses of the system are compared. For the experimental results, the converter has been used as a rectifier connected to a resistive load on the dc side, as shown in Fig. 7. On the ac side the converter is connected to a three phase VARIAC via three single phase transformers. The parameters of the system are summarised in TABLE IV. Due to the large amount of waveforms to be recorded simultaneously, two different acquisition methods have been used. The results are recorded via the use of oscilloscopes and the master control board. Two oscilloscopes have been used to measure: (1) the L-CH voltages, the T-CH voltages, (2) the dc voltage and the (3) voltages on the blocking capacitors $C_{T}$. The first oscilloscope is a YOKOGAVA DCM2024 with $200 \mathrm{MHz}$ bandwidth and $2.5 \mathrm{GSa} / \mathrm{s}$ sample rate, and the second is Tektronix DP0 2024 with the same bandwidth and $1 \mathrm{GSa} / \mathrm{s}$ sample rate.

In addition, the master board stores the converter side ac voltages/currents, the SM voltages and the energy/current transients. The waveforms recorded via the master are sampled at $2 \mathrm{kHz}-$ a quarter of the switching frequency $(8 \mathrm{kHz})$.

The parameters of the control loops adopted have been obtained employing standard linear control design tools and are given in TABLE V. The notch filters included in the table are designed to reduce the voltage ripple on the feedback of the SM voltages of the T-CHs. The transfer function of the adopted filter is,

$$
H(s)=\frac{s^{2}+\omega^{2}}{s^{2}+\frac{\omega_{0}^{2}}{Q} s+\omega_{0}}
$$

where $\omega_{0}$ is the frequency to be cancelled and $\mathrm{Q}$ is the quality factor of the filter.

\section{A. Steady state converter operation}

These tests are mainly needed to verify if the controllers drive the system state variables to the reference values. In Fig. 20 the voltage produced by the three L-CHs are shown.

TABLE IV: System Parameters

\begin{tabular}{lll}
\hline \multicolumn{1}{c}{ Symbol } & \multicolumn{1}{c}{ Quantity } & Value \\
& & \\
$V_{d c}$ & dc voltage & $450 \mathrm{~V}$ \\
$V_{L L}$ & line to line voltage & $415 \mathrm{~V}$ \\
$f_{g}$ & line frequency & $50 \mathrm{~Hz}$ \\
$r$ & transformer turns ratio & $90 / 240$ \\
$L_{s}$ & line inductance & $1.7 \mathrm{mH}$ \\
$R_{S}$ & line resistance & $0.3 \Omega$ \\
$L_{d c}$ & dc inductance & $11.7 \mathrm{mH}$ \\
$R_{L O A D}$ & Load resistance & $120 \Omega$ \\
$N_{S M} M_{L-C H}$ & number of SMs for each L-CH \\
$N_{S M} M_{T-C H}$ & number of SMs for each T-CH \\
$V_{S M}$ & SM nominal voltage & 3 \\
$C_{S M}$ & SM capacitance & $60 \mathrm{~V}$ \\
$R_{S M}$ & SM resistance & $5 \mathrm{mF}$ \\
$C_{T}$ & blocking capacitance & $39.1 \mathrm{k} \Omega$ \\
$f_{P W M}$ & PWM frequency & $880 \mathrm{uF}$ \\
$f_{S O R T}$ & sorting frequency & $8 \mathrm{kHz}$ \\
& & $2 \mathrm{kHz}$ \\
\hline \hline
\end{tabular}


Fig. 21 shows the waveforms produced by the three T-CHs. Even though each T-CH is able to produce 7 voltage levels only three levels are used in this operating condition. This is due to the fact that the amplitude of the voltage produced by this chain-link is directly proportional to the phase currents, and in this experiment the currents are below the nominal value. The appearance of extra levels in this figure is due to very short "error" pulses created by an interaction of the bridge dead-time delay and cycling of the zero state between upper and lower device pairs in each bridge to equalise losses. Due to their short duration, they have an insignificant effect on the overall operation. The voltage drops across the blocking capacitors $C_{T}$ are presented in Fig. 22. The small differences in ac amplitude are mainly due to mismatches in the blocking capacitor values $C_{T}$. The currents flowing in the ac side of the converter are shown in Fig. 24. The small distortion present in the current waveforms can be justified by the non-compensated dead times and by the fact that the network voltages feeding the VARIAC have some distortion.

Fig. 25 presents the SM voltages including both the L$\mathrm{CH}$ and T-CH SMs. As expected, the SM working voltages match the reference value $V_{S M}^{r e f}=60 \mathrm{~V}$. The L-CH SMs have a fundamental voltage ripple at $50 \mathrm{~Hz}$, while, as expected, the T-CH SM voltages have a fundamental ripple at $100 \mathrm{~Hz}$. The peak to peak voltage fluctuations are smaller in the T-CHs than in the L-CHs. Clearly, the T-CHs could have a smaller capacitance for the same peak to peak voltage ripple as the L-CHs. However, the same value of $C_{S M}$ is used in both to unify and simplify the design.

TABLE V: Parameters of the implemented control loops

\begin{tabular}{|c|c|c|c|}
\hline Symbol & Bandwidth $[\mathrm{Hz}]$ & Parameter & Value \\
\hline TEC L-CH & $\approx 3.5$ & $k_{p}$ & 0.1 \\
\hline$\overline{\mathrm{T}} \overline{\mathrm{EC}} \overline{\mathrm{T}}-\overline{\mathrm{C}} \overline{\mathrm{H}}$ & $--\bar{\approx} 3 . \overline{.5}$ & $-\frac{k_{i}}{\overline{k_{p}}}-$ & $-\frac{1.0}{0.1}$ \\
\hline$\overline{\mathrm{IP}} \overline{\mathrm{C}}---$ & $-\bar{\approx} 0 . \overline{3} 5^{-}$ & $\frac{k_{i}}{\overline{k_{p}}}-$ & $-\frac{1.0}{0.1}$ \\
\hline$\overline{\mathrm{P}} \mathrm{L} \overline{\mathrm{L}}^{---}$ & $--\approx \overline{3}-$ & $\frac{k_{i}}{\overline{k_{p}}}-$ & $-\frac{0.1}{0.2}$ \\
\hline$\overline{\mathrm{P}}+\overline{\mathrm{R}}^{---}$ & 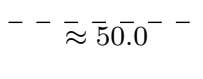 & $\begin{array}{c}-\frac{T_{i}}{k_{p}}- \\
k_{R}\end{array}$ & $\begin{array}{r}0.05 \\
-0.1 \\
0.1\end{array}$ \\
\hline$\overline{\mathrm{T}}-\overline{\mathrm{C}} \mathrm{H}$ notch & - & $\begin{array}{c}\omega_{c} \\
--\frac{\omega_{c}}{\bar{\omega}_{0}}- \\
Q\end{array}$ & $\begin{array}{r}1.0 \\
-\quad 2 \pi 50 \\
2 \pi \overline{1} 0 \overline{0} \\
10.0\end{array}$ \\
\hline
\end{tabular}

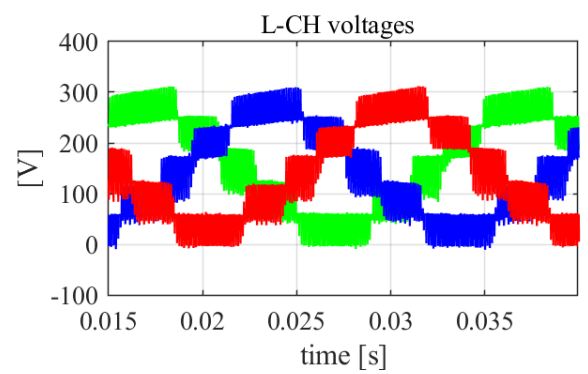

Fig. 20: Voltage waveforms produced by the L-CHs.

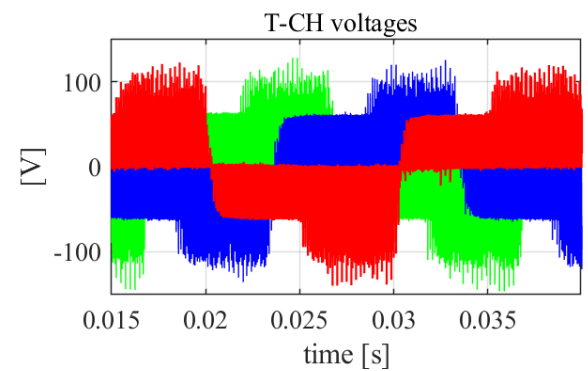

Fig. 21: Voltage waveforms produced by the T-CHs.

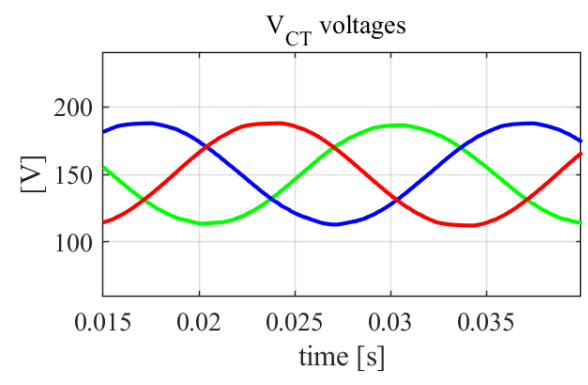

Fig. 22: Blocking capacitor $C_{T}$ voltages.

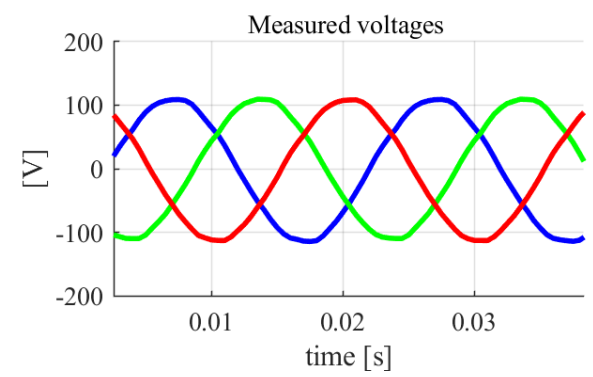

Fig. 23: Ac voltages. The currents are measured on the converter side of the transformer.

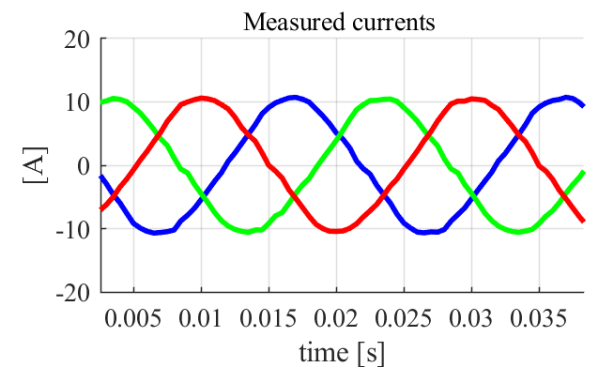

Fig. 24: Ac currents. The currents are measured on the converter side of the transformer.

\section{B. Reactive reference variation}

To test the functionality of the current control loops, independently from the dynamics of the energy control, a 6 A amplitude step in the reactive component of the current is imposed. The dc load during this test is $R_{L O A D}=90 \Omega$. The dynamic response of the ac current control loops is shown in Fig. 26. This figure also shows a corresponding simulation result, illustrating the match between the modelling and the experimental results.

The phase current takes approximately one fundamental 


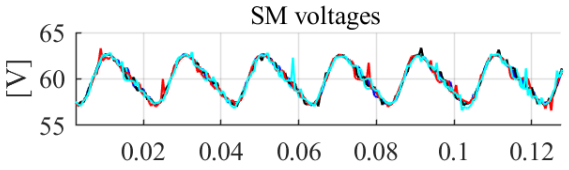

(a)

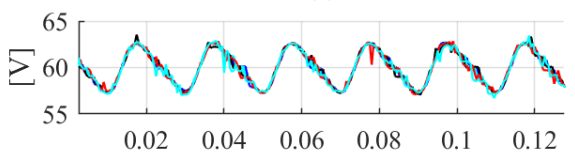

(b)

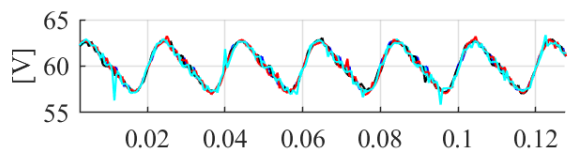

(c)

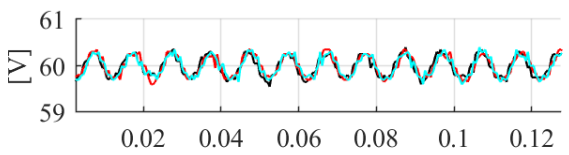

(d)

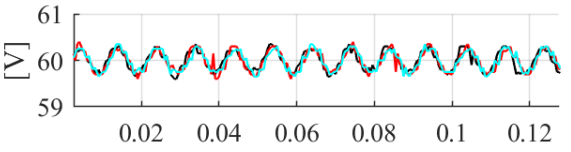

(e)

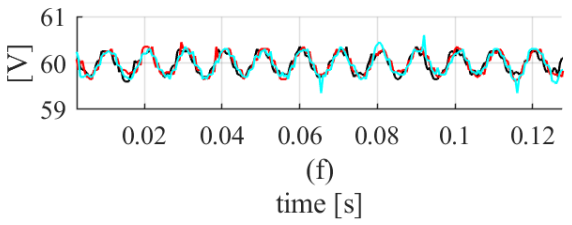

Fig. 25: SM voltages of the converter chain-links: (a) L-CH SM voltages of the 1st phase, (b) L-CH voltages of the 2 nd phase, (c) L-CH voltages of the 3rd phase, (d) T-CH SM voltages of the 1st phase, (e) T-CH voltages of the 2 nd phase, (f) T-CH voltages of the 3rd phase. The colours used to mark the SMs of the L-CHs are respectively: blue, green, black, red and cyan. The colours used to mark the SMs of the T-CHs are respectively: black, red and cyan.

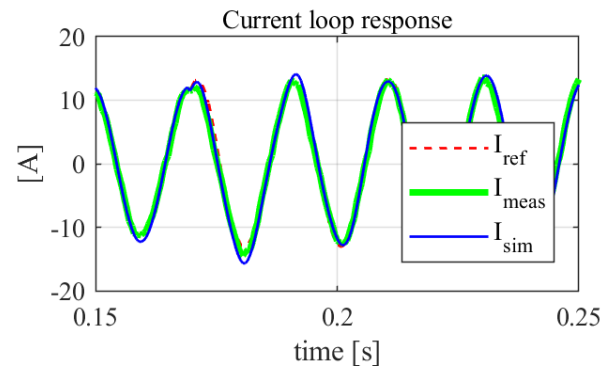

Fig. 26: Dynamic response of the current control loop to a variation of the reactive component set point.

period to settle.

\section{Energy variation}

In order to validate the dynamic response of the the TEC, a $5 \%$ energy reference step was applied. In Fig. 27 the dynamics of the total energy controller are shown. The settling time

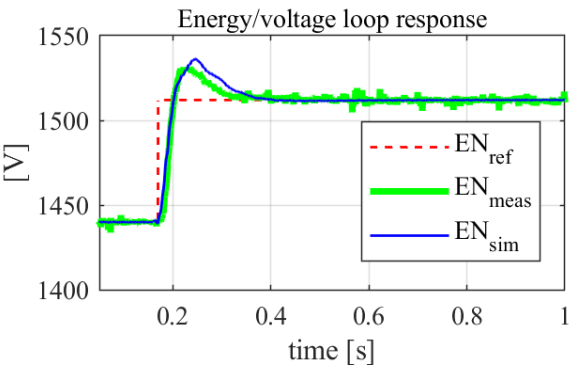

Fig. 27: Dynamic response of the total energy/voltage control loop to a variation of the energy/voltage reference value.

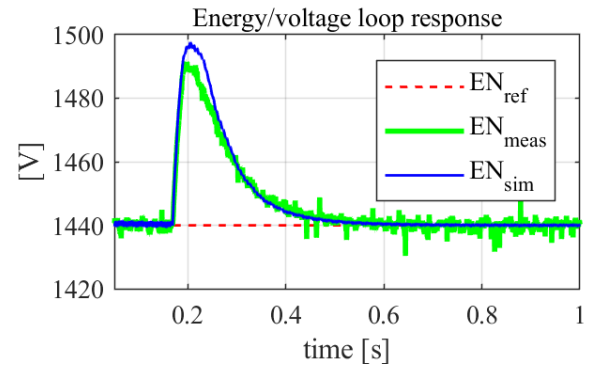

Fig. 28: Dynamic response of the energy/voltage control loop to load variation.

of the energy loop is approximately $0.3 \mathrm{~s}$ according to the design bandwidth of the TEC loops. This result confirms the effectiveness of the analytical design and again the match between modelling and experiment is illustrated.

\section{D. $32 \%$ Load variation}

To further verify the TEC response to perturbations, a $32 \%$ load variation has been applied. Initially the converter operates with a dc load $R_{L O A D}$ of $60 \Omega$, requiring an active power flow from the grid to the dc side of $3.3 \mathrm{~kW}$. After a certain period the network is switched to a resistor of $90 \Omega$, for an equivalent power flow of $2.25 \mathrm{~kW}$. Despite the different load conditions the controllers are still tuned according to TABLE V. The response of the TEC is shown in Fig. 28. After the load step, the system requires approximately $0.3 \mathrm{~s}$ to settle as expected according to the bandwidth of the TEC. A good match between the experimental results and the simulation model is demonstrated again.

\section{CONCLUSION}

An innovative modular multilevel Series Chain-link Converter (SCC) has been presented. The proposed solution takes advantage of the inherent property of a symmetric set of voltages in order to reduce the required number of SMs and to achieve a more concentrated energy storage than in a traditional MMC. As such, it is expected to be interesting where converter footprint is at a premium, for example, in off-shore applications. The blocking capacitor $C_{T}$ fulfils the task of removing the dc offset produced by the corresponding $\mathrm{L}-\mathrm{CH}$, and a T-CH is adopted "to cancel" the ac voltage drop across the blocking capacitors and also to provide an additional degree of freedom to deal with different converter operating 
conditions. The converter has the potential to reduce the number of required SMs by $60 \%$ while maintaining approximately the same energy storage of an equivalent MMC. In addition, the number of passive elements are reduced since the converter needs only three blocking capacitors. The experimental results from a laboratory scale prototype prove the viability of this new solution.

\section{ACKNOWLEDGEMENT}

The authors would like to acknowledge the contribution made to the early stages of this work by their late colleague Dr Colin Oates.

\section{APPENDIX}

The minimum number of half bridge SMs for the L-CHs can be evaluated according to:

$$
N_{S M_{L-C H}}=\left\lceil\left\lceil\sqrt{\frac{2}{3}} \frac{\left(\frac{\left(1+R M_{a c}\right) \cdot\left(2-R M_{d c}\right)}{1-R M_{d c}}\right) r \cdot V_{L L}}{V_{S M}}\right\rceil \cdot k_{r}\right\rceil
$$

The minimum number of full bridge SMs of the T-CHs in the active configuration can be evaluated according to:

$$
N_{S M_{T-C H}}^{\text {hybrid }}=\left\lceil\left\lceil\frac{\sqrt{6}}{5} \cdot \frac{\left(1+R M_{a c}\right) \cdot r \cdot V_{L L}}{V_{S M}}\right\rceil \cdot k_{r}\right\rceil
$$

The minimum number of half bridge SMs of the T-CHs in the hybrid configuration can be evaluated according to:

$$
N_{S M_{T-C H}}^{\text {active }}=\left\lceil\left\lceil\sqrt{\frac{2}{3}} \frac{\frac{\left(1+R M_{a c}\right) \cdot\left(1+R M_{d c}\right)}{1-R M_{d c}} \cdot r \cdot V_{L L}}{V_{S M}}\right\rceil \cdot k_{r}\right\rceil
$$

The energy fluctuation in the T-CH SMs of the Hybrid configuration can be evaluated by,

$$
\begin{aligned}
E(t)= & \int \hat{V}_{T-C H}^{i} \sin \left(\omega_{g} \cdot t+\frac{\pi}{2}-\varphi-\frac{i-1}{3} \pi\right) \\
& \cdot \frac{1}{r} \hat{I}_{\text {phase }}^{i} \sin \left(\omega_{g} \cdot t-\varphi-\frac{i-1}{3} \pi\right) d t, i=1,2,3
\end{aligned}
$$

where $\hat{V}_{T-C H}^{i}$ is assumed to approximately $V_{d c} / 6$. With a similar approach the the energy fluctuation in the T-CH SMs of the Active configuration can be calculated by,

$$
E(t)=\int \frac{V_{d c}}{3} \cdot \frac{1}{r} \hat{I}_{\text {phase }}^{i} \sin \left(\omega_{g} \cdot t-\varphi-\frac{i-1}{3} \pi\right) d t
$$

\section{REFERENCES}

[1] Y. Shakweh and E. A. Lewis, "Assessment of medium voltage pwm vsi topologies for multi-megawatt variable speed drive applications," in Power Electronics Specialists Conference, 1999. PESC 99. 30th Annual IEEE, vol. 2, pp. 965-971. IEEE, 1999.

[2] H. Abu-Rub, S. Bayhan, S. Moinoddin, M. Malinowski, and J. Guzinski, "Medium-voltage drives: Challenges and existing technology," IEEE Power Electronics Magazine, vol. 3, no. 2, pp. 29-41, 2016.

[3] B. R. Andersen, L. Xu, P. J. Horton, and P. Cartwright, "Topologies for VSC transmission," Power Engineering Journal, vol. 16, no. 3, pp. 142-150, Jun. 2002.
[4] A. Nabae, I. Takahashi, and H. Akagi, "A new neutral-point-clamped pwm inverter," IEEE Trans. Ind. Appl., vol. IA-17, no. 5, pp. 518-523, Sep. 1981

[5] M. Marchesoni, M. Mazzucchelli, and S. Tenconi, "A non conventional power converter for plasma stabilization," in Power Electronics Specialists Conference, 1988. PESC '88 Record., 19th Annual IEEE, pp. 122-129 vol.1, Apr. 1988.

[6] T. Meynard and H. Foch, "Multi-level conversion: high voltage choppers and voltage-source inverters," in Power Electronics Specialists Conference, 1992. PESC '92 Record., 23rd Annual IEEE, pp. 397-403 vol.1, Jun. 1992.

[7] J. D. Ainsworth, M. Davies, P. J. Fitz, K. E. Owen, and D. R. Trainer, "Static VAr compensator (statcom) based on single-phase chain circuit converters," IEE Proceedings - Generation, Transmission and Distribution, vol. 145, no. 4, pp. 381-386, Jul. 1998.

[8] A. Lesnicar and R. Marquardt, "An innovative modular multilevel converter topology suitable for a wide power range," in Power Tech Conference Proceedings, 2003 IEEE Bologna, vol. 3, pp. 6 pp. Vol.3-, Jun. 2003.

[9] J. Gerdes, "Siemens debuts HVDC PLUS with san francisco's trans bay cable," Living Energy, vol. 5, pp. 28-31, 2011.

[10] P. L. Francos, S. S. Verdugo, H. F. Álvarez, S. Guyomarch, and J. Loncle, "INELFE-Europe's first integrated onshore HVDC interconnection," in Power and Energy Society General Meeting, 2012 IEEE, pp. 1-8. IEEE, 2012.

[11] P. Bresesti, W. L. Kling, R. L. Hendriks, and R. Vailati, "HVDC connection of offshore wind farms to the transmission system," IEEE Transactions on Energy Conversion, vol. 22, no. 1, pp. 37-43, Mar. 2007.

[12] M. Hiller, D. Krug, R. Sommer, and S. Rohner, "A new highly modular medium voltage converter topology for industrial drive applications," in Power Electronics and Applications, 2009. EPE'09. 13th European Conference on, pp. 1-10. IEEE, 2009.

[13] R. Feldman, M. Tomasini, J. Clare, P. Wheeler, D. Trainer, and R. Whitehouse, "A hybrid voltage source converter arrangement for HVDC power transmission and reactive power compensation," in Power Electronics, Machines and Drives (PEMD 2010), 5th IET International Conference on, pp. 1-6, Apr. 2010.

[14] E. Amankwah, A. Costabeber, A. Watson, D. Trainer, O. Jasim, J. Chivite-Zabalza, and J. Clare, "The series bridge converter (SBC): A hybrid modular multilevel converter for HVDC applications," in 2016 18th European Conference on Power Electronics and Applications (EPE'16 ECCE Europe), pp. 1-9, Sep. 2016.

[15] M. M. C. Merlin, T. C. Green, P. D. Mitcheson, D. R. Trainer, R. Critchley, W. Crookes, and F. Hassan, "The alternate arm converter: A new hybrid multilevel converter with dc-fault blocking capability," IEEE Transactions on Power Delivery, vol. 29, no. 1, pp. 310-317, Feb. 2014.

[16] G. P. Adam, B. Alajmi, K. H. Ahmed, S. J. Finney, and B. W. Williams, "New flying capacitor multilevel converter," in 2011 IEEE International Symposium on Industrial Electronics, pp. 335-339, Jun. 2011.

[17] Q. Hao, B. T. Ooi, F. Gao, C. Wang, and N. Li, "Three-phase seriesconnected modular multilevel converter for HVDC application," IEEE Transactions on Power Delivery, vol. 31, no. 1, pp. 50-58, Feb. 2016.

[18] S. Bai, Y. Meng, B. Liu, H. Zhang, and H. Ma, "Modeling and control of a novel three-phase series-connected modular multilevel converter in T configuration," in 2016 IEEE PES Asia-Pacific Power and Energy Engineering Conference (APPEEC), pp. 1322-1326, Oct. 2016.

[19] S. Allebrod, R. Hamerski, and R. Marquardt, "New transformerless, scalable modular multilevel converters for HVDC-transmission," in 2008 IEEE Power Electronics Specialists Conference, pp. 174-179. IEEE, 2008.

[20] K. Friedrich, "Modern HVDC plus application of vsc in modular multilevel converter topology," in 2010 IEEE International Symposium on Industrial Electronics, pp. 3807-3810, Jul. 2010.

[21] A. Junyent-Ferré, P. Clemow, M. M. C. Merlin, and T. C. Green, "Operation of HVDC modular multilevel converters under dc pole imbalances," in 2014 16th European Conference on Power Electronics and Applications, pp. 1-10, Aug. 2014.

[22] D. Jovcic and K. Ahmed, High voltage direct current transmission: converters, systems and dc grids. John Wiley \& Sons, 2015.

[23] C. Oates, "Modular multilevel converter design for vsc HVDC applications," IEEE Journal of Emerging and Selected Topics in Power Electronics, vol. 3, no. 2, pp. 505-515, Jun. 2015.

[24] F. Tardelli, A. Costabeber, and R. Feldman, "A voltage source converter," Patent EP3 352 354A1, 2017. 
[25] F. Tardelli, "A series chain-link modular multilevel converter (SCC) for HVDC applications," Ph.D. dissertation, The University of Nottingham, 2018.

[26] H. Fujita, S. Tominaga, and H. Akagi, "Analysis and design of a dc voltage-controlled static var compensator using quad-series voltagesource inverters," IEEE Trans. Ind. Appl., vol. 32, no. 4, pp. 970-978, Jul. 1996.

[27] M. Zygmanowski, B. Grzesik, and R. Nalepa, "Capacitance and inductance selection of the modular multilevel converter," in Power Electronics and Applications (EPE), 2013 15th European Conference on, pp. 1-10, Sep. 2013.

[28] E. M. Farr, R. Feldman, J. C. Clare, A. J. Watson, and P. W. Wheeler "The alternate arm converter (aac) - "short-overlap" mode operationanalysis and design parameter selection," IEEE Transactions on Power Electronics, vol. 33, no. 7, pp. 5641-5659, 2018.

[29] M. M. C. Merlin and T. C. Green, "Cell capacitor sizing in multilevel converters: cases of the modular multilevel converter and alternate arm converter," IET Power Electronics, vol. 8, no. 3, pp. 350-360, 2015.

[30] R. Teodorescu, M. Liserre, and P. Rodriguez, Grid Synchronization in SinglePhase Power Converters. IEEE, 2007. [Online]. Available: https://ieeexplore.ieee.org/document/5732930

[31] R. Teodorescu, F. Blaabjerg, M. Liserre, and P. C. Loh, "Proportionalresonant controllers and filters for grid-connected voltage-source converters," IEE Proceedings - Electric Power Applications, vol. 153, no. 5, pp. 750-762, Sep. 2006.

[32] M. Saeedifard and R. Iravani, "Dynamic performance of a modular multilevel back-to-back HVDC system," IEEE Transactions on Power Delivery, vol. 25, no. 4, pp. 2903-2912, Oct. 2010.

[33] K. Sharifabadi, L. Harnefors, H.-P. Nee, S. Norrga, and R. Teodorescu, Design, control, and application of modular multilevel converters for HVDC transmission systems. John Wiley \& Sons, 2016.

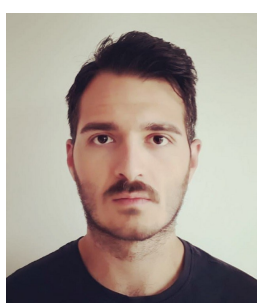

Francesco Tardelli received his master's degree in electrical engineering from the University of Pisa (Italy) in 2014, and the $\mathrm{PhD}$ in electrical and electronic engineering from the University of Nottingham (UK) in 2018. From 2018-2019 he was a postdoctoral research fellow in the Power Electronics, Machines and Control Group at The University of Nottingham. In 2019 he joined Amantys Power Electronics Limited (part of the group Maschinenfabrik Reinhausen $\mathrm{GmbH}$ ) and he is currently working on Modular Multilevel Converters for MV applications. His research interests include modular multilevel converter topologies, control systems applied to power conversion and control and modelling of power converters.

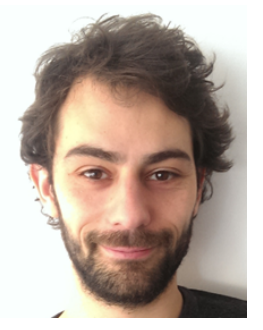

Alessandro Costabeber received the MSc degree with honours in Electronic Engineering from the University of Padova, Italy, in 2008 and the Ph.D. in Information Engineering from the same university in 2012, on energy efficient architectures and control for future residential microgrids. In 2014 he joined the PEMC group at the University of Nottingham, UK as Lecturer in Power Electronics. Since September 2019 he has been Associate Professor at the University of Padova, Italy. His current research interests include modular multilevel converters for HVDC, high power density converters, control and stability analysis of AC and DC microgrids. Dr. Costabeber received the IEEE Joseph John Suozzi INTELEC Fellowship Award in Power Electronics in 2011.

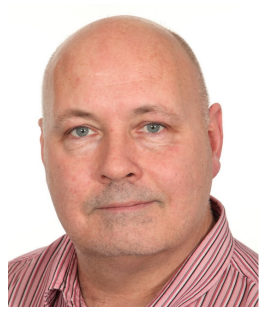

David Trainer received his BSc (Hons) and $\mathrm{PhD}$ degrees from Staffordshire Polytechnic, UK, in 1985 and 1991, respectively. Between 1985 and 1998 he worked for GEC on the development of advanced thyristor valves for HVDC power transmission and later pioneered the use of GTO based modular multilevel converter (M2C) technology for reactive power compensation. In 1998, David joined Goodrich working on the development of electric actuation systems for civil and defence aerospace. In 2004 he joined Rolls-Royce to support more electric engine development in the strategic research centre. In 2008, David joined Alstom to work on the development of high-power voltage source converters for HVDC power transmission leading the development of new converter technologies. In 2018, David re-joined Rolls-Royce as the global technology lead for power electronics and is focussing mainly on aerospace and marine electric propulsion systems and electric starter-generators. He is an Honorary Professor of electrical power conversion at The University of Nottingham, UK.

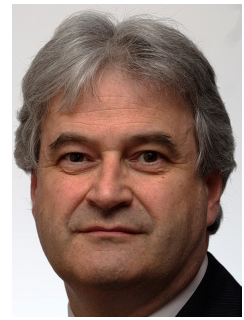

Jon Clare was born in Bristol, UK, in 1957. He received the $\mathrm{BSc}$ and $\mathrm{PhD}$ degrees in electrical engineering from the University of Bristol, Bristol, UK, in 1979 and 1990, respectively. From 1984 to 1990, he was a Research Assistant and Lecturer with the University of Bristol, where he was involved in teaching and research on power electronic systems. Since 1990, he has been with the Faculty of Engineering at the University of Nottingham, UK. He is currently Professor of Power Electronics and is the Head of the Electrical and Electronic Engineering Department. He is a member of the Power Electronics, Machines and Control Research Group at Nottingham. His research interests are in power-electronic converters and their applications and control. Jon Clare is the recipient of a Royal Society Wolfson Research Merit Award. 